\title{
Mathematical model and numerical calculations of disastrous pressure phenomena in rock mass with weakening cavity
}

\author{
Sergey Lavrikov* \\ Chinakal Institute of Mining SB RAS, Novosibirsk, 630091, Russia
}

\begin{abstract}
The study focuses on deformation and stability of structurally nonuniform rock masses in the vicinity of a mined-out void. Rock masses can for a long time accumulate energy of external forces as internal stresses. The accumulated energy can release both as aseismic relaxation of stresses and as rock bumps and rock bursts. The numerical analysis uses the rock mass model including self-balancing stresses and strength loss. The paper gives examples of calculations of standard problems in mining and illustrates feasibility of modeling disastrous pressure phenomena.
\end{abstract}

\section{Introduction}

Modern geomechanics is developing along the path of increasingly complex mathematical modeling of rocks and rock masses. Recent approaches aim to provide an adequate description of complicated structural hierarchy of rocks. The block structure and pores filled with weaker rocks or fluids need that mathematical models include the nonlinear poroelastic properties of rocks, anisotropy and possible loss of strength. For another thing, an evolving nonuniform rock mass can accumulate elastic energy in the form of internal self-balancing stresses, and some areas of rock mass can represent potential sources of energy. This fact is proved by field observations and lab-scale experiments [1-6]. Any impact (for instance, mining activities) can trigger and provoke unbalance in areas of high concentration of self-balancing stresses. Subject to certain conditions, such unbalance can be accompanied with aseismic relaxation of stresses, or can result in disastrous phenomena induced by lithostatic pressure, such as seismic activity, rock bumps and rock bursts [7-9]. Some mining-induced dynamic pressure phenomena are described in [10-12].

Mathematical modeling and computational methods develop in different ways. Rock masses are described using a damage index [13], or by nonlocal models with structural parameters [14], or assuming the structure as a property of the space of deformation [15]. With advance in computer engineering, the discrete element method (DEM) enjoys a wider application in the last decades. In DEM the rock mass structure is represented by a set of discrete particles, and deformation is reduced to a relative motion of the particles with regard to various interaction potentials of the particles $[16,17]$. However, one of the most popular approaches to modeling in structurally nonuniform geomechanics is yet continuum models with internal variables [18-20]. The internal variables are additionally introduced to

\footnotetext{
*Corresponding author: 1vk64@mail.ru
} 
describe the behavior of grains, blocks and pore media; conditions at fracture surfaces and grain boundaries; fluid permeation parameters, etc., and enable characterization of wide range properties governed by the internal structure of rock mass. Analogous models are used in related fields of mechanics, for instance, in description of grain structure of metals [21].

This study considers the problem on modeling of dynamic phenomena induced by strata pressure in rock mass in the vicinity of underground openings using a plane model of structurally nonuniform rock as an active environment with inner sources and sinks of energy [22, 23].

\section{Mathematical model}

Within the framework of the approach presented in [23], a continuum model of structurally nonuniform rocks is constructed as follows. A representative volume of sufficient number of grains (blocks) is selected. The initial random packing is replaced by a well-formed effective packing composed of equally sized grains arranged at the points of a square lattice (plane strain). In this fashion, the unit volume represents a framework of grains with a periodic structure. It is assumed that grains can slide past each other. The pore space is filled with a cementing material. Thus, shearing of grains can induce compression of the pore fill and the related accumulation of elastic energy. This approach allows describing such properties of a unit volume as dilatancy, internal friction, plasticity and anisotropy. Furthermore, inclusion of nonlinearity in shearing of grains makes it possible to take into account the ability to accumulate (at the stage of strengthening) and release (at the stage of strength loss) elastic energy.

According to [23], the force and kinematics of the unit volume are described not by initial discontinuities but using averaged continuous microstress tensor fields for grains $t$ (or $\tau$ in rotated coordinate system) and pores $p$, as well as averaged microstrain tensors fields for grains $\varepsilon^{t}$ (or $\varepsilon^{\tau}$ ), pores $\varepsilon^{p}$ and relative grain-boundary sliding $\varepsilon^{R}$. The introduced values are connected by compatibility conditions which permit finding averaged tensors of stresses $\sigma^{*}$ and strains $\varepsilon^{*}$ in a unit volume in the coordinates of a preset periodic lattice of grains:

$$
\begin{aligned}
\sigma_{i j}^{*} & =t_{i j}+2 m p_{i j}=t_{i j}+2(1-m) \tau_{i j}, \\
\varepsilon_{i j}^{*} & =\varepsilon_{i j}^{t}+\varepsilon_{i j}^{R}=(1-m) \varepsilon_{i j}^{\tau}+m \varepsilon_{i j}^{p},
\end{aligned} \quad i, j=1,2
$$

where $0<m<1$ characterizes clear opening rate. Relations (1) mean that a certain volume is in constant stress state of grains and pore material (the tensors $t_{i j}, p_{i j}$ are determined), and the macro volume stresses $\sigma_{i j}^{*}$ are then calculated unambiguously. The inverse problem allows many solutions to be obtained. It is possible that different-sign and mutually compensatory non-zero stresses act in grains and in pores, and total macrostresses are zero, i.e. $t_{i j} \neq 0, p_{i j} \neq 0, \sigma_{i j}^{*}=0$. In this case, when solving boundary problems on macrostress state in rock mass, it is required to determine distribution of these stresses in structural components, i.e., it is necessary to describe concentration zones (if any) of internal selfbalancing stresses.

We express properties of structural components in terms of the matrix equalities $t=T^{t} \cdot \varepsilon^{t}, \tau=T^{\tau} \cdot \varepsilon^{\tau}, p=P \cdot \varepsilon^{p}, t=R \cdot \varepsilon^{R}$, including the matrices of specific properties 
of grains $\left(T^{t}, T^{\tau}\right)$, pores $(P)$ and grain interaction $(R)$. In this case, conditions (1) allow formulating constitutive relations for a continuum (averaged) unit volume in the form:

$$
\left(\begin{array}{l}
\varepsilon_{11} \\
\varepsilon_{22} \\
\varepsilon_{12}
\end{array}\right)=W \cdot\left(\left(T^{t}+R\right)^{-1}+2\left(T^{\tau}+P\right)^{-1}\right)^{-1} \cdot W^{-1} \cdot\left(\begin{array}{c}
\sigma_{11} \\
\sigma_{22} \\
\sigma_{12}
\end{array}\right),
$$

where $\sigma_{i j}, \varepsilon_{i j}$ are the averaged tensors of stresses and strains in the unit volume in arbitrary coordinates $O x_{1} x_{2}$; the matrix $W=W(\alpha)$ characterizes rotation of the wellformed effective grain packing by an angle $\alpha$ relative to the coordinates $O x_{1} x_{2}$.

Constitutive relations (2) together with the Cauchy relations and equations of equilibrium constitute a closed system. This system is solved numerically using the finite element method (FEM). The nonlinearity is included in terms of variability of the coefficients in (2), which are the functions of the stress state. In this case, the initial closed system should be re-formulated in terms of increments (the system is linear for the increments), and the general solution should be obtained in a quasistatic formulation, by loading steps with regard to varying properties of rock mass.

Accumulation and release of energy is modeled by setting a nonlinear diagram of boundary sliding of grains with regard to stages of strengthening and strength loss. The slope of the strength loss curve is critical: when the actual slope is higher than the critical slope, the accumulated energy releases as jumps of strength loss. Evidently, it is impossible to model dynamic phenomena within the framework of the quasistatic formulation. The algorithmic approach in [24] introduces an artificial external force at the moment of a jump, which restrains the jump and enables the accumulated energy to 'bleed' in the mode of relaxation. As a result, the contact shears acquire the same increment as in the dynamic problem without regard to inertia. This approach makes it possible to obtain a satisfactory approximation of the problem solution with regard to the strength loss jumps.

The model described in [25-27] was used to solve some boundary problems with regard to different properties of rocks, and to describe the rock mass property to accumulate and release elastic energy. Modeling of unstable deformation with regard to internal selfbalancing stresses concentrated in a finite area in rock mass in the vicinity of an arch crosssection tunnel was for the first time undertaken in [28]. The type of the system of equations was analyzed, and it was shown that a post-limit deformation modulus could induce the jump-wise release of elastic energy. Some other examples of numerical calculations are given below.

\section{Formulation of boundary value problems}

Let us consider two standard geomechanical problems on deformation of rock mass in the vicinity of a mined-out void. Figures 1 and 2 depict the computational domains in the vicinity of a circular cross-section opening (the first problem) and in the vicinity of a stoping front (the second problem). The initial stresses (superscript is the number of loading step) are selected to be distributed linearly:

$$
\sigma_{22}^{0}=-\gamma\left(H-x_{2}\right), \quad \sigma_{11}^{0}=\lambda \sigma_{22}^{0}, \quad \sigma_{12}^{0}=0,
$$

where $H$ is the occurrence depth of the opening; $\lambda$ is the lateral earth pressure coefficient; $\gamma$ is the overlying rock weight. It is assumed that initial (intact) rock mass is subjected to 
mining operations which form an underground opening. Loading reduces to decreasing normal and shear stresses at the opening boundary $\Gamma_{0}$ (Figs. 1 and 2). The boundary conditions for one incremental step take the form: $\left.\Delta \sigma_{n}\right|_{\Gamma_{0}}=-\left.\Delta d^{k} \cdot \sigma_{n}^{0}\right|_{\Gamma_{0}},\left.\Delta \tau_{n}\right|_{\Gamma_{0}}=-\left.\Delta d^{k} \cdot \tau_{n}^{0}\right|_{\Gamma_{0}},\left.\Delta \sigma_{n}\right|_{\Gamma \backslash \Gamma_{0}}=0,\left.\Delta u_{n}\right|_{\Gamma \backslash \Gamma_{0}}=0$, where $0 \leq \Delta d^{k} \leq 1$ is the loading parameter (load released from the boundary), $k$ is the loading step. Total relaxation of the boundary from stresses conforms with the condition: $d=\sum_{k} \Delta d^{k}=1$.

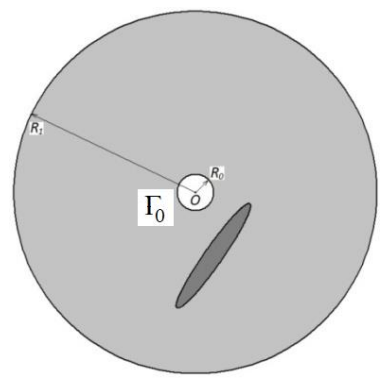

Fig. 1. Computational domain of rock mass in the vicinity of circular cross-section opening

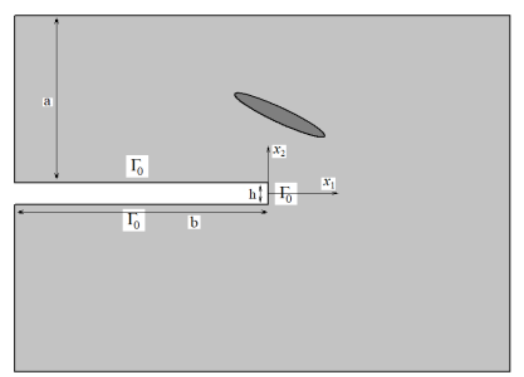

Fig. 2. Computational domain of rock mass in the vicinity of stoping front

According to (1), we need to find distribution of initial macrostresses (3) between components of the internal structure. Let:

$$
\begin{aligned}
& t_{11}^{0}=0.5 \sigma_{11}^{0^{*}}, p_{11}^{0}=0.5 \sigma_{11}^{0 *} /(2 m), \\
& t_{22}^{0}=0.5 \sigma_{22}^{0 *}, p_{22}^{0}=0.5 \sigma_{22}^{0 *} /(2 m), \\
& t_{12}^{0}=0.5 \sigma_{12}^{0^{*}}+\delta \tau^{\max }, p_{12}^{0}=\left(0.5 \sigma_{12}^{0^{*}}-\delta \tau^{\max }\right) /(2 m),
\end{aligned}
$$

where $\delta$ is a dimensionless parameter. It is seen that the normal stresses are equally distributed in grains and pores (hereinafter, it is assumed that $m=0.5$ ). The shear stress distribution depends on $\delta$. When $\delta=0$ the shear load features uniform distribution in grains and pores. At $\delta \neq 0$ the shear load distribution is nonuniform. When $\delta=1$ the shear load of grains becomes critical (reaches the value $\tau^{\max }$ ) and any minor extra loading results in jump-wise loss of strength.

Let rock mass in the vicinity of a mined-out void contain a high-concentration zone of self-balancing stresses, i.e., the zone of concentration of different-sign and mutually compensatory shear stresses. The zone has a shape of an elongated ellipse (the darkfield domains in Figs. 1 and 2). Let us consider the calculation results obtained at $\delta=0$ everywhere in the computation domain and at $\delta=0.8$ (i.e., critical) inside the higher stress concentration zone.

\section{Calculation data}

Figures $3 \mathrm{a}$ and $3 \mathrm{~b}$ present the calculation data for the two problems with post-limit values of deformation modulus at grain contacts. The curves illustrate the static equilibrium behavior with the increasing loading parameter $d$. The maximum values are $d \approx 0.38$ in the first problem and $d \approx 0.86$ in the second problem. Put it otherwise, the boundary of the 
mined-out void in rock mass in the condition of stable equilibrium is never unloaded. Release of stresses at the boundary leads to such loading redistribution in the adjacent rock mass zone that the internal self-balancing stresses lose equilibrium and the jump of strength loss takes place. Further relaxation of the void boundary from stresses results in a series of such jumps, and, finally, the parameter $d$ passes over zero and assumes a negative value:: $d \approx-0.57$ at the point $D$ in the first problem (Fig. 3a) and $d \approx-0.03$ at the point $H$ in the second problem (Fig. 3b). This means that equilibrium of rock mass is only possible if the reaction pressure of the mine support at the void boundary exceeds the lithostatic pressure of intact rock mass. Obviously, without additional measures aimed to suppress release of energy of internal stresses, the mined-out opening can lose stability and fail.

a)

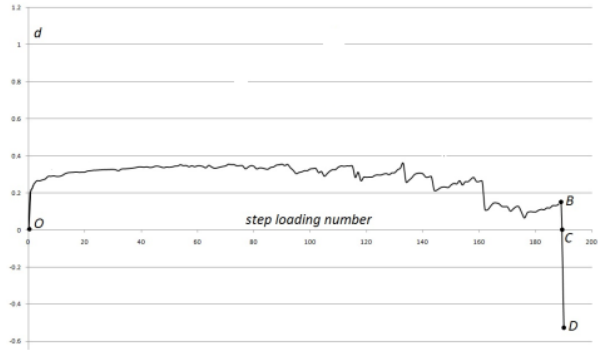

b)

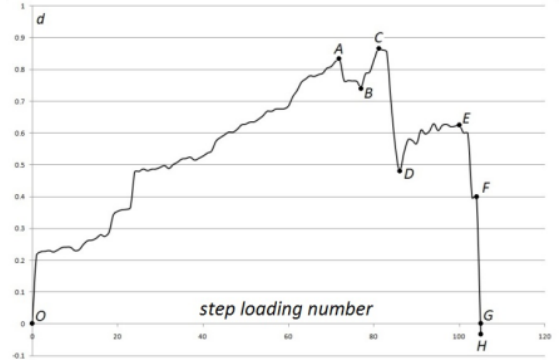

Fig. 3. Change of parameter $d$ (reduction in stress at mined-out void boundary relative to initial value): a) the first problem; $b$ ) the second problem

Figure 4 shows the calculated contour lines of the maximum shear stress $\tau_{\max }=0.5 \sqrt{\left(\sigma_{11}-\sigma_{22}\right)^{2}+4 \sigma_{12}^{2}}$ and maximum shear $\gamma_{\max }=\sqrt{\left(\varepsilon_{11}-\varepsilon_{22}\right)^{2}+4 \varepsilon_{12}^{2}}$ in the first problem, and Fig. 5 presents the same characteristics obtained in the second problem.

a)

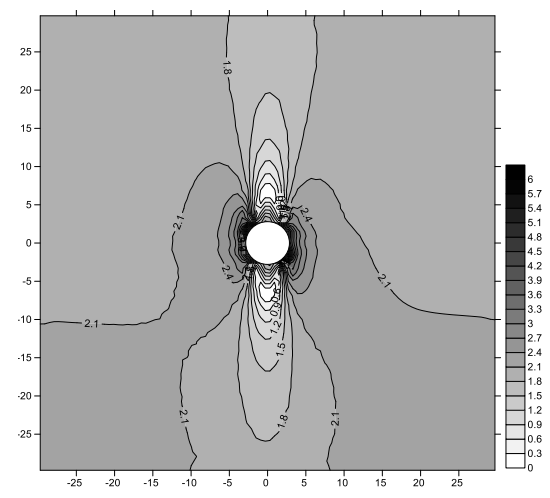

b)

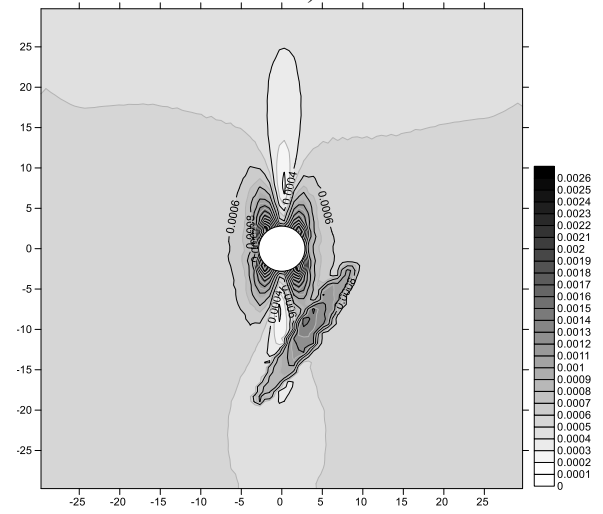

Fig. 4. Theoretical contour lines of stress-strain behavior in rock mass in loading at $d \approx 0.18$ (point $B$ in Fig. 3a): a) $\tau_{\max }$; b) $\gamma_{\max }$

It is seen that inside the high concentration zone of self-balancing stresses, high-rate shearing takes place and leads to asymmetry of the stress state (Fig. 4a). On the whole, the release of energy of self-balancing stresses leads to essential degradation of strength in rock 
mass in the vicinity of the mined-out void and, as a consequence, to a great drop in its loadbearing capacity.

a)

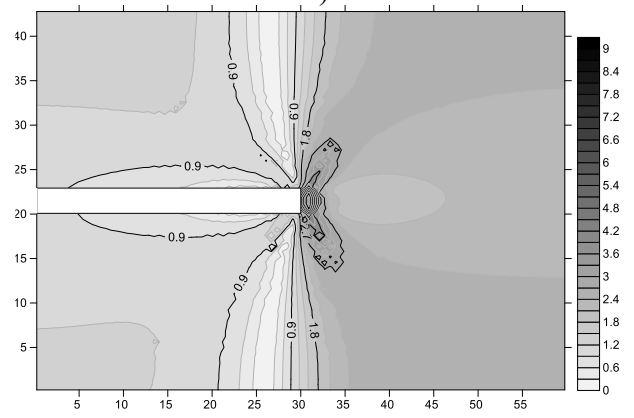

б)

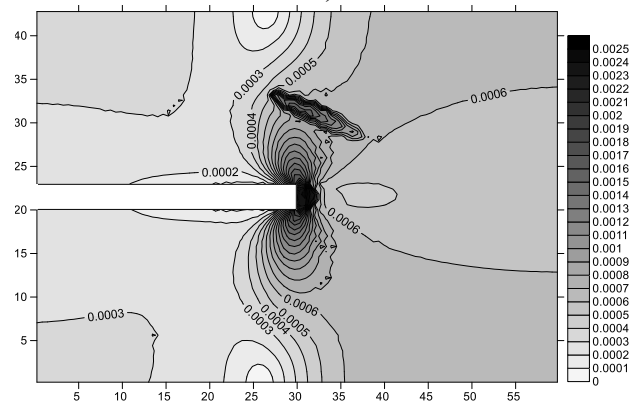

Fig. 5. Theoretical contour lines of stress-strain behavior in rock mass in loading at $d \approx 0.4$ (point $F$ in Fig. 3b): a) $\tau_{\max }$; b) $\gamma_{\max }$

\section{Conclusions}

1. The mathematical model and FEM-based algorithm discussed in this paper allow modeling the post-limit stress-strain behavior with regard to jumps of strength loss in rock mass.

2. The presence of concentration zones of high internal stresses in rock mass in the vicinity of the mining operation front can provoke dynamic pressure phenomena.

The study was carried out within the framework of the Basic Research Project, Registration No. $121052500138-4$.

\section{References}

1. V.S. Ponomarev, Geotektonika, 2, 66-75 (2011)

2. A.N. Stavrogin, O.A. Shirkes, J. of Mining Science, 22 (4), 235-244 (1986)

3. P.M. Goryainov, I.V. Davidenko, Doklady Akad. Nauk SSSR, 247 (5), 1212-1215 (1979)

4. K.T. Tazhibaev, D.K. Tazhibaev, Geomechanics in Mining: Conference Proceedings, 17-27 (Yekaterinburg, 4-5 June, 2014)

5. S.V. Lavrikov, A.F. Revuzhenko, J. of Mining Science, 27 (4), 288-293 (1991)

6. A.P. Bobryakov, V.P. Kosykh, A.F. Revuzhenko, J. of Mining Science, 51 (1), 10-16 (2015)

7. G.A. Sobolev, A.V. Ponomarev, Physics of earthquakes and foreshocks (Nauka, Moscow, 2003)

8. V.G. Bykov, Physical Mesomechanics, 23, 271-278 (2020)

9. S. Das, C.H. Scholz, Bull. Seismol. Soc. Amer.,71, 1669-1675 (1983)

10. V.V. Adushkin, S.B. Turuntaev, Induced and triggered seismicity (IDG RAN, Moscow, 2015)

11. G.G. Kocharyan, S.B. Kishkina, J. of Mining Science, 54 (5), 744-750 (2018)

12. G.R. Foulger, M.P. Wilson, et al., Earth-Science Reviews, 178, 438-514 (2018) 
13. I.Yu. Smolin, P.V. Makarov, et al., Physical Mesomechanics, 21, 297-304 (2018)

14. A.F. Revuzhenko, O.A. Mikenina, J. of Appl. Mech. and Tech. Phys., 59, 332-340 (2018)

15. A.F. Revuzhenko, J. of Mining Science, 52(5), 842-850 (2016)

16. V.I. Klishin, A.V. Nikolaev, S.V. Klishin, IOP Conf. Ser.: EES, 377, (2019)

17. S.V. Klishin, S.V. Lavrikov, et al., IOP Conf. Ser.: J. of Physics, 973 (2018)

18. W. Wu, J. Lin, X. Wang, Acta Geotechnica, 12 (6), 1373-1382 (2017)

19. D. Kolymbas, I. Herle, P.A. Wolffersdorff, Int. J. of Num. and Anal. Meth. in Geomechanics, 19, 415-436 (1995)

20. G.M. Eggert, P.R. Dawson, Int. J. of Mechanical Sciences, 29 (2), 95-113 (1987)

21. V.V. Novozhilov, Yu.I. Kadashevich, Microstresses in engineering materials (Mashinostroenie, Leningrad, 1990)

22. A.F. Revuzhenko, J. of Mining Science, 26 (4) , 301-308 (1990)

23. A.F. Revuzhenko, Mechanics of elastoplastic media and nonstandard analysis (NGU, Novosibirsk, 2000)

24. S.V. Lavrikov, A.F. Revuzhenko, J. of Mining Science, 26 (6), 485-892 (1990)

25. S.V. Lavrikov, J. of Mining Science 46 (5) 485-494 (2010)

26. S.V. Lavrikov, Fizich. Mezomekhanika 13 (4) 53-63 (2010)

27. S.V. Lavrikov, A.F. Revuzhenko, Desiderata Geotechnica, ed. W. Wu, 79-85 (2019)

28. S.V. Lavrikov, A.F. Revuzhenko, J. of Mining Science, 56 (6), 887-902 (2020) 